\title{
The Quantum Oscillatory Modulated Potential I-The Hydrogen Atom
}

\author{
Waldemar Wolney Filho \\ Instituto de Física, Universidade Federal de Goiás, Goiânia, Brazil \\ Email: wwolney@yahoo.com.br
}

Received April 25, 2012; revised May 22, 2012; accepted June 15, 2012

\begin{abstract}
In this work we are presenting a modified Coulomb potential function to describe the interaction between two microscopic electric charges. In particular, concerning the interaction between the proton and the electron in the hydrogen atom. The modified potential function is the product of the classical Coulomb potential and an oscillatory function dependent on a quantized phase factor. The oscillatory function picks up only selected points along the Coulomb potential, creating potential wells and barriers around the nucleus of the atom. The new potential reveals us new features of the hydrogen atom. Searching for a manner to determine the phase factor, we are using the concept of the de Broglie particle wavelike behavior and the quantum analogue of the virial theorem for describing the bound motion of a particle in a central force field. This procedure is a kind of feedback action, where we are making use of well established concepts of the quantum mechanics aiming to determine the phase factor of the new interaction potential.
\end{abstract}

Keywords: Potential; Atom; Electron; Oscillator Fuction

\section{Introduction}

This work is the first of a series of four papers, for which we are revisiting, reexamining and questioning some points about the foundations of the atomic theory developed, mostly between the years 1910-1930, by many distinguished physicists: Niels Bohr, Erwin Schroedinger, Werner Heisenberg, Alberl Einstein, Max Born, Louis de Broglie and many others. What motivate us to produce this work are, in our opinion, some unclear and questionable points, that we believe still exist, particularly, concerning the applicability of the classical Coulomb potential for describing the interaction between two microscopic electric particles. For instance, in the hydrogen atom, the Coulomb potential, used by Bohr and, years later, in the quantum mechanics (Schroedinger) to describe many of the known properties of the current atomic model, is an increasing smooth function of the distance between the proton and the electron. Being so, how can the electron possibly know that certain positions around the nucleus of the atom are more likely to be occupied than others? [1] What is the real cause for this behavior for the electron? It is difficult to imagine the electron making regular probabilistic choice to occupy certain particular positions in the vicinity of the nucleus of the atom, based solely in the characteristics presented by the Coulomb potential. The observed discrete values of the total energy of the electron, suggest us the existence of potential wells and barriers in the form of closed shells surrounding the nucleus of the atom. The question of how and why the electron is able to jump from one state of equilibrium to another, emitting or receiving energy radiation (light), could be better explained if we can accept the idea that the electron is tunnelling through these potential barriers.

The stability of the atom is an interesting question that deserves further explanation. ${ }^{1}$ The de Broglie wavelength associated to the waving nature of a microscopic charged particle is an attribute of the mass, as postulated by Louis de Broglie in 1923, or is it an attribute of the charge of the particle? ${ }^{2}$ Another interesting question is concerned to the actual movement of the electron around the nucleus of the atom. Is the electron orbiting the nucleus of the atom as predicted by Bohr theory? Is it behaving like a cloud of negative charge around the nucleus of the atom? Or yet is it oscillating radially in quantum wells like a harmonic oscillator?

The Coulomb potential belongs to what is known as classical physics. Since long ago it is a fact well known that it correctly describes the energy interaction between two punctual macroscopic electric charges. Also, it is

\footnotetext{
${ }^{1}$ The stability of the atom will be treated in the second paper of this series.

${ }^{2}$ This subject will be considered in the third paper and, for an uncharged particle, possessing an electric dipole moment, the subject will treated in the fourth paper of the series.
} 
well known that the behavior of a particle in the micro-world of the quantum physics is not the same as that in the macro-world of classical physics. What we want to say is that, the classical Coulomb potential reveal us only the macro-behavior of the interaction between electric particles This make us to suspect the existence of a different interaction potential, capable of describing more accurately the physics of the atoms, and also be able to answer the questions formulated above. We believe that the choice of any potential function that proposes to be candidate to describe the interaction between two microscopic charges, in some way, needs to carry by itself the seeds of the quantum physics of the atom, that is, the quantization of the angular momentum and energy, both concepts, intimately related to the Heisenberg uncertainty principle. Besides, in some way, the new potential must be responsible to the wavelike nature of the particles.

\section{The Hydrogen Atom}

In this paper, trying to answer the questions formulated above, let us postulate that in the hydrogen atom, the interaction energy between the proton (nucleus of the atom) and the electron, considered as spinless particles, can be expressed by the product of the Coulomb potential and one oscillatory function, written as

$$
U_{\text {osc. }}(r)=-\frac{1}{4 \pi \epsilon_{0}} \frac{Z e^{2}}{r} \cos ^{2}(\xi r) .
$$

The parameter $\xi$, with dimension of inverse of distance, represents a radial-space frequency. We believe that the phase factor $\xi r$ of the oscillatory function is the key point to answer the questions formulated preously. As we will see soon, this phase factor is directly related to the Heisenberg uncertainty principle and the quantization of the angular momentum.

For fixed values of the parameter $\xi(\xi \neq 0)$, the function $\cos ^{2}(\xi r)$ oscillates between zero and one, while the function $U_{\text {osc. }}(r)$ oscillates between zero and the classical Coulomb potential energy. The Equation (1), tell us that when $\xi \rightarrow 0, \cos ^{2}(\xi r) \rightarrow 1$, the new potential function becomes equal to the classical Coulomb potential.

$$
U_{\text {сои. }}(r)=-\frac{1}{4 \pi \in_{0}} \frac{Z e^{2}}{r} .
$$

However, we need to observe that if we make $\xi=0$, we have $U_{\text {osc. }}(r)=U_{\text {cou. }}(r)$ for all values of the distance $r$ between the proton and the electron in the hydrogen atom. This implies that the linear momentum of the electron $p=\hbar \xi$ is null. That is, the electron is at rest in the LFR (Laboratory Frame of Reference). This condition strongly contraries one of the basilar principles of the quantum mechanics, the Heisenberg uncertainty principle $\Delta r \Delta p_{r} \geq \hbar$. That is, if we make the uncertainty of the linear momentum $\Delta p_{r}=\hbar \Delta \xi=0$, we have $\Delta r \rightarrow \infty$, and the radial position of the electron becomes completely undetermined. On the other hand, if we make $\Delta r=0$, we have $\Delta p_{r}=\infty$, making the kinetic energy of the electron infinitely large [2-4]. This clearly shows the inadequacy of the Coulomb potential to describe the interaction between the proton and the electron in the hydrogen atom, except at particular points of the space in the vicinity of the nucleus of the atom. The phase factor of the oscillatory function selects only specific points of the Coulomb potential, making it to satisfy the Heisenberg uncertainty principle only at points, where the condition $\xi r=n \pi$ is satisfied. This constitutes a quantized relation between the normal and the reciprocal space.

Looking for the determination of the parameter $\xi$, we will make use of the quantum-mechanical analogue of the virial theorem and the de Broglie relation between the linear momentum of the particle and the wave number $k$. With this procedure, we are making use of well known quantum concepts to determine the phase factor of the new potential. The quantum analogue of the virial theorem can be set in the form [4]

$$
\langle T\rangle=\frac{1}{2}\langle\mathbf{r} \cdot \nabla U(r)\rangle
$$

and the required de Broglie relation is

$$
p=\hbar k
$$

To facilitate the application of Equation (1), it turns out to be convenient that the parameter $\xi$ may be expressed in terms of known quantities such as, the charge and mass of the electron and some physical constants. With the use of Equations (1) and (3), the average kinetic energy of the electron in the hydrogen atom is found to be expressible as

$$
\left\langle\frac{p^{2}}{2 m}\right\rangle=\frac{1}{2}\left(\frac{Z e^{2}}{4 \pi \epsilon_{0}}\right)\left\langle\left[\frac{1}{r} \cos ^{2}(\xi r)+\xi \sin (2 \xi r)\right]\right\rangle
$$

With the use of Equation (4) it follows that

$$
\langle k\rangle=\left[\left(\frac{1}{4 \pi \epsilon_{0}}\right) \frac{Z m e^{2}}{\hbar^{2}}\right]^{\frac{1}{2}}\left\langle\left[\frac{1}{r} \cos ^{2}(\xi r)+\xi \sin (2 \xi r)\right]^{\frac{1}{2}}\right\rangle .
$$

We will select the desired particular points along the Coulomb potential, observing that every time the product $\xi r$ satisfies the quantization relation,

$$
\xi r=n \pi, n=1,2,3, \cdots,
$$

the Coulomb potential becomes equal to the oscillatory potential and the Equation (6), may be written as 


$$
\frac{\langle k\rangle}{n}=\left[\frac{1}{4 \pi \epsilon_{0}} \frac{Z m e^{2}}{\hbar^{2} n^{2}}\right]^{\frac{1}{2}}\left\langle\left(\frac{1}{r}\right)\right\rangle^{\frac{1}{2}},
$$

or

$$
\frac{\langle k\rangle}{n} r=\left[\frac{1}{4 \pi \epsilon_{0}} \frac{Z m e^{2}}{\hbar^{2} n^{2}}\right]^{\frac{1}{2}}\left\langle\left(\frac{1}{r}\right)\right\rangle^{\frac{1}{2}} r .
$$

The Equations (7) and (9) become consistent if we set $\xi=\langle k\rangle / n$ and

$$
\left\langle\left(\frac{1}{r}\right)\right\rangle=\left[\left(\frac{1}{4 \pi \epsilon_{0}}\right) \frac{Z m e^{2}}{\hbar^{2} n^{2}}\right],
$$

what permit us to rewrite the Equation (9) as

$$
\langle\xi\rangle r=\left\langle\left[\frac{1}{4 \pi \in_{0}} \frac{\pi Z m e^{2}}{\hbar^{2} n^{2}}\right]\right\rangle r .
$$

Comparing the Equations (7) and (11) we can identify the space-frequency $\xi$ as a quantized quantity given by

$$
\left\langle\xi_{n}\right\rangle=\frac{1}{4 \pi \epsilon_{0}} \frac{\pi Z m e^{2}}{n^{2} \hbar^{2}} .
$$

In the hydrogen atom we have $Z=1$ and, in its ground state, $n=1$. The quantity $\left(4 \pi \in_{0} \hbar^{2} / \mathrm{me}^{2}\right)$ may be identified as the first Bohr radius $\left\langle r_{1}\right\rangle=a_{0}=0.529 \AA$ of the electron. The Equation (12) may be rewritten as

$$
\left\langle\xi_{n}\right\rangle=\frac{\pi}{n^{2} a_{0}}
$$

This implies that for two atomic bound particles, such as the proton and the electron in the hydrogen atom, the average value of the linear momentum of the electron is also a quantized quantity

$$
\left\langle p_{n}\right\rangle=\left(\frac{1}{4 \pi \epsilon_{0}}\right) \frac{m e^{2}}{n \hbar} .
$$

Using Equation (13), the new potential function may be rewritten as

$$
U_{n}(r)=-\frac{1}{4 \pi \epsilon_{0}} \frac{e^{2}}{r} \cos ^{2}\left(\xi_{n} r\right) .
$$

We are naming this potential by Quantum Oscillatory Modulated Potential-QOMP; because, it is quantized, it oscillates with k-space frequency $\xi_{n}$, and it is partially modulated by the classical Coulomb potential. Using the condition given by Equation (7) and the Equations (1) and (5), the average total energy of the electron with respect to the nucleus is

$$
\begin{aligned}
\langle T+U\rangle & =\left\langle\frac{1}{2}\left(\frac{1}{4 \pi \epsilon_{0}}\right) e^{2} \frac{1}{r}-\frac{1}{4 \pi \epsilon_{0}} Z e^{2} \frac{1}{r}\right\rangle \\
& =-\frac{1}{2}\left(\frac{1}{4 \pi \epsilon_{0}}\right) Z e^{2}\left\langle\frac{1}{r}\right\rangle .
\end{aligned}
$$

Taking the average value $\langle 1 / r\rangle$, as given by Equation (10), the energy of the electron becomes quantized and may be written as

$$
E_{n}=-\frac{1}{2}\left(\frac{1}{4 \pi \epsilon_{0}}\right)^{2} \frac{m e^{4}}{n^{2} \hbar^{2}},
$$

as would be expected, it is the same relation as obtained with Bohr theory and also with the solution of the time-independent Schroedinger wave equation for the hydrogen atom when the Coulomb potential is used. But, differently from these two theories, that in different circumstances, make explicit use of the quantization of the angular momentum to obtain the total energy of the electron, in this work, the same result is achieved without making explicit use of this concept. Implicitly, the quantization of the angular momentum is contained in the phase factor of the oscillatory function, Equation (7).

The Figure 1 shows a graphic of the potential $U_{1}(r)$. As shown in the figure, the electron in the hydrogen atom occupies the first well with position and total energy satisfying the Equations (10) and (17), respectively. The Figure 2 shows the graphics of $U_{n}(r)$ for $n=1,2$ and 3 . The values of the energies shown in the graphic determined by the positions of the electron are, respectively, $-13.6 \mathrm{eV},-3.4 \mathrm{eV}$ and $-1.5 \mathrm{eV}$. The same results are predicted by Equation $(17)[4,5]$.

The new potential present us with several interesting features: 1) Its waving character shows a sequence of periodic wells and barriers with variable depth and height; 2) The first well is at the position most likely to be occupied by the electron in the ground state of the atom; 3) The quantization of the energy may be attributed to the existence of concentric quantum shells, formed by wells and barriers, created by the interaction of the nucleus of the atom and the electron; 4) According to this potential, the electron bound to the nucleus can only jumps from one quantum well to another by tunneling through finite potential barriers. The tunneling effect (a purely quantum mechanical phenomenon) now appears naturally as necessary condition for emission or absorption of energy (light) by the atom; 5) Another new interesting aspect of the oscillatory potential, observed in the Figure 2, and predicted by Equations (13) and (14) is the fact that the de Broglie wavelength associated to the electron is equal to the first Bohr radius of the electron in the hydrogen atom. Thus, the average distance between the proton and the electron, for any excited state of the atom, may be written as

$$
\left\langle r_{n}\right\rangle=n^{2}\left\langle r_{1}\right\rangle .
$$

Or, equivalently, in terms of the de Broglie wavelengths we may write

$$
\left\langle\lambda_{n}\right\rangle=n^{2}\left\langle\lambda_{1}\right\rangle
$$




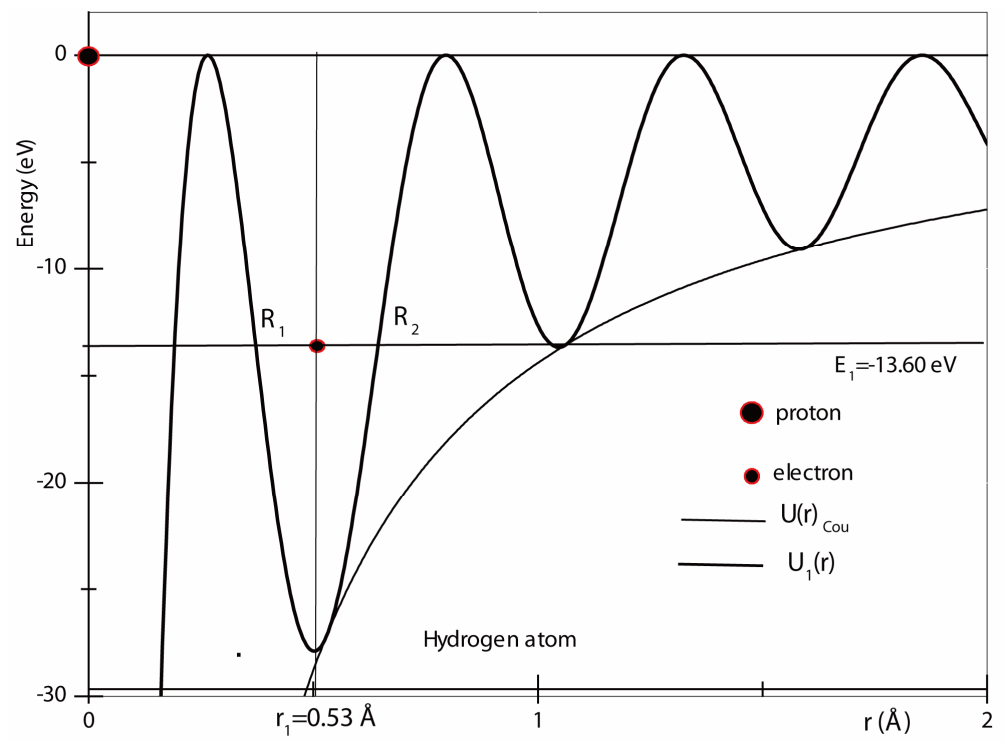

Figure 1. The Coulomb potential $U(r)_{\text {Coul }}$ and the quantum oscillatory modulated potential $U_{1}(r)$, showing the position and energy of the electron in the ground state of the hydrogen atom. The positions $R_{1}$ and $R_{2}$ are classical turning points for the electron.

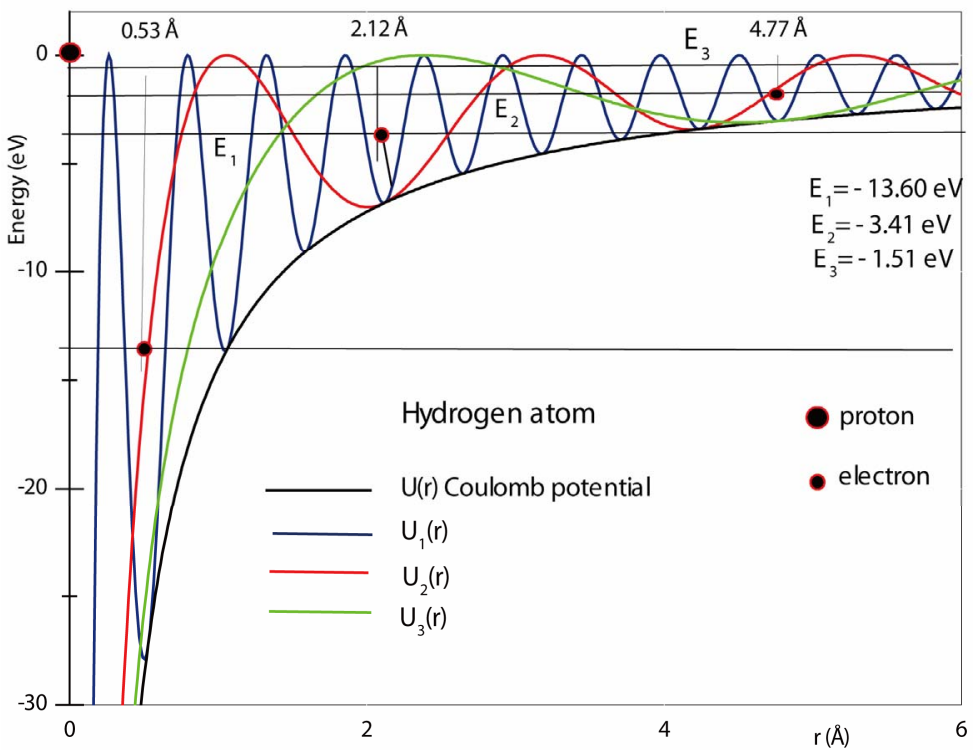

Figure 2. The Coulomb potentials $U(r)_{C o u l}$, the quantum oscillatory modulated potentials $U_{n}(r)$ and the energy levels and positions of the electron in the hydrogen atom for $n=1,2$ and 3.

This result is completely different from that predicted by the quantization rule $2 \pi r=n \lambda$ for each allowed orbit for the electron, as postulated by Louis de Broglie, to explain the quantization of the angular momentum of the electron in the Old Quantum Theory [5,6].

Figure 2 shows that the averaged positions occupied by the electron in the first three quantum wells of $U_{1}(r)$, $U_{2}(r)$ and $U_{3}(r)$ are, respectively, $\left\langle r_{1}\right\rangle=0.53 \AA$, $\left\langle r_{2}\right\rangle=4\left\langle r_{1}\right\rangle$ and $\left\langle r_{3}\right\rangle=9\left\langle r_{1}\right\rangle$. In this work we are arguing that the Coulomb potential is not totally appropriated to describe correctly the potential interaction between two microscopic electric charges. However, as is well known, the solution of the time independent Schroedinger wave equation predicts correctly the quantization of the angular momentum and energy of the electron, when Coulomb potential is used in the Hamiltonian of the protonelectron system. This occur because, in the solution of the radial part of the Schroedinger differential wave equation, without noticing it, we are making use the quantization rule $\xi r=n \pi$ for the phase factor of the 
oscillatory function of the new potential. That is, implicitly, we are making $\cos ^{2}\left(\xi_{n} r\right)=1$ and, consequently, selecting only the points of minima of the new potential, where both interaction potentials are coincident, see Figure 2. In these selected points the Coulomb potential satisfies the Heisenberg uncertainty principle. In terms of a mathematical treatment, the quantization of the phase factor $\xi r$ allow us to see that the Coulomb potential is a particular case of the oscillatory potential, by transforming the Schroedinger radial differential equation

$$
\begin{aligned}
& \frac{1}{\rho^{2}} \frac{\mathrm{d}^{2} S(\rho)}{\mathrm{d} \rho^{2}}+\frac{2}{\rho} \frac{\mathrm{d} S(\rho)}{\mathrm{d} \rho} \\
& +\left[\frac{\delta}{\rho} \cos ^{2}\left(\xi_{n} \frac{1}{2 \beta} \rho\right)-\frac{l(l+1)}{\rho^{2}}-\frac{1}{4}\right] S(\rho)=0,
\end{aligned}
$$

obtained with the use of the Quantum Oscillatory Modulated Potential in the Hamiltonian of the protonelectron system into the simpler and familiar radial differential equation $[4,5]$

$$
\frac{1}{\rho^{2}} \frac{\mathrm{d}^{2} S(\rho)}{\mathrm{d} \rho^{2}}+\frac{2}{\rho} \frac{\mathrm{d} S(\rho)}{\mathrm{d} \rho}+\left[\frac{\delta}{\rho}-\frac{l(l+1)}{\rho^{2}}-\frac{1}{4}\right] S(\rho)=0,
$$

obtained with the use of the classical Coulomb potential, with the condition $\xi_{n} \rho / 2 \beta=n \pi$ or $\cos ^{2}\left(\xi_{n} \rho / 2 \beta\right)=1$. The solution of Equation (21) is well known and it is found in many books of modern physics literature [4-7]. Its solution shows that the total energy of the electron in the hydrogen atom is the same as predicted by Equation (17). Up to this point we can also state that the Coulomb potential is restricted to describe correctly the interaction between two microscopic electric charges only at particular points of the space around nucleus of the atom, points where the quantization relation $\xi_{n} r=n \pi$ and, consequently, the Heisenberg uncertainty principle are satisfied.

\section{A New Electric Interaction Microscopic Force}

The electric force between two macroscopic electric point charges $Q_{1}$ and $Q_{2}$, separated by a distance $r$, is given by the well known classical Coulomb formula

$$
F_{\text {coul. }}= \pm \frac{1}{4 \pi \in_{0}} \frac{Q_{1} Q_{2}}{r^{2}}
$$

which is perfectly valid as far as we consider the two charges built up of a very large number of protons or electrons, but still considered as point charges. However, the electric force between two microscopic charges such as proton-proton, proton-electron, or electron-electron, originated from the new potential function, has the form of a wave packet, written as

$$
\begin{aligned}
F_{n}(r) & =-\frac{\mathrm{d}}{\mathrm{d} r} U_{n}(r) \\
& = \pm \frac{1}{4 \pi \epsilon_{0}} Z e^{2}\left[\frac{1}{r^{2}} \cos ^{2}\left(\xi_{n} r\right)+\frac{\xi_{n}}{r} \sin \left(2 \xi_{n} r\right)\right],
\end{aligned}
$$

The forces $F_{n}(r)$ oscillate with the same $k$-space frequencies $\xi_{n}$. For the hydrogen atom, Figure 3 shows a graphic of $F_{1}(r)$, as predicted by Equation (23). Since this force is the gradient of the energy function $U_{1}(r)$, which presents a minimum at position $\left\langle r_{1}\right\rangle$, it becomes null at this point. We must observe that with the use of the classical Coulomb potential this situation never occurs, the interaction force between the proton and the electron is always attractive. The acceleration due to the electric force being null at this position, eliminates the argument or defiance against the Classical Laws of the Electrodynamic that an electron revolving the nucleus of the atom, lose energy by radiation [8-10]. The force being null at the equilibrium position $\left\langle r_{1}\right\rangle=a_{0}$, means that the electron cannot be orbiting the nucleus of the atom as predicted by Bohr theory. Thus, according to the QOMP, the angular momentum of the electron in its ground state is null, in agreement with the prediction of the Schroedinger radial differential wave Equation (21). On the other hand, the electron cannot be a static physical object due to the Heinsenberg uncertainty principle. The angular moment of the electron being null' implies that the movement of the electron, inside a quantum well must be radial, bouncing back and forth, confined by two potential barriers.

This argument is reinforced by observing that in Figure 3 , the interaction force between the proton and the electron is repulsive to the left of the position $r_{1}$ and attractive to the right of this position. That is, the electron is always being pushed towards the equilibrium position, acting like a harmonic oscillator. However, being accelerated towards the equilibrium position, again, the electron should lose energy by radiation and the problem of stability of the atom continues to be unexplained. The stability of the atom will be object of a deeper discussion in the second paper of this series of work. Figure 4 shows the behavior of the electric forces $F_{n}(r)$ for $n=1,2$ and 3 . The graphics show that the waving character of interaction electric force decreases when $n$ increases, in agreement with the Bohr correspondence principle. The reasons for this behavior are two: 1) The destructive interference of the waving pattern of the individual charges, protons and electrons occur because, when macroscopic charges ${ }^{3}$ are built up, these particles do not occupy the same position in space and the waving pattern of the force must be destroyed by an interference process; 2) The actual measurements of the interaction

${ }^{3} \mathrm{~A}$ macroscopic charge of $1 \mu \mathrm{C}=1 \times 10^{-6} \mathrm{C}$, contains about $10^{13}$ electronic charges. 


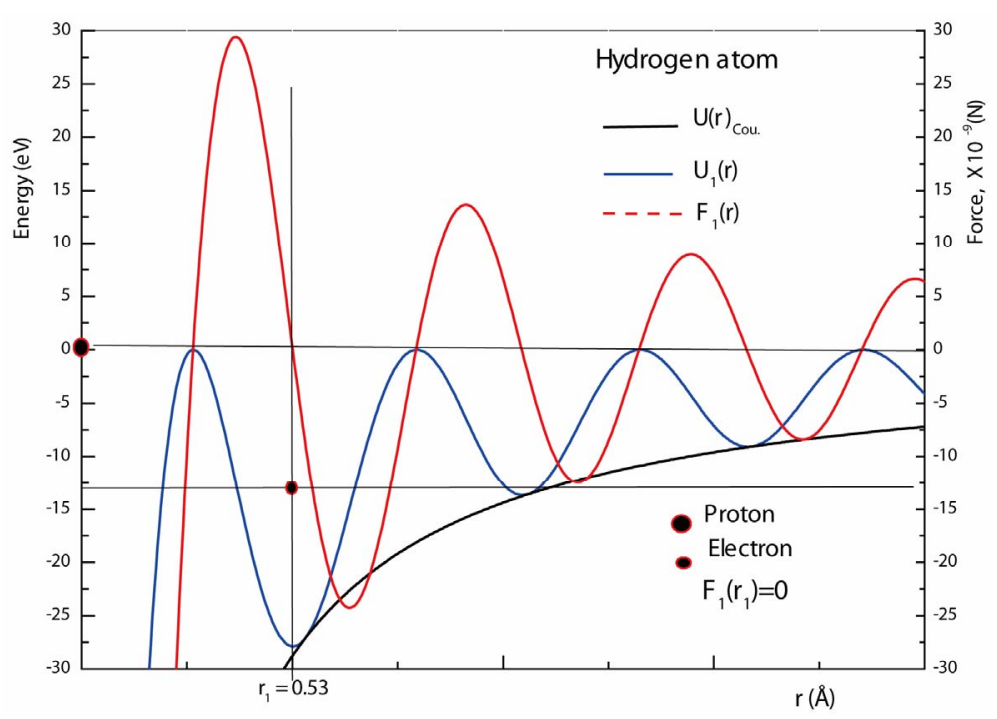

Figure 3. The interaction oscillatory force $F_{1}(r)$, the quantum modulated potential $U_{1}(r)$ and the Coulomb potential $U(r)_{C o u l}$ in the ground state of the hydrogen atom. The figure also shows that the force is null at the equilibrium position $r_{1}$.

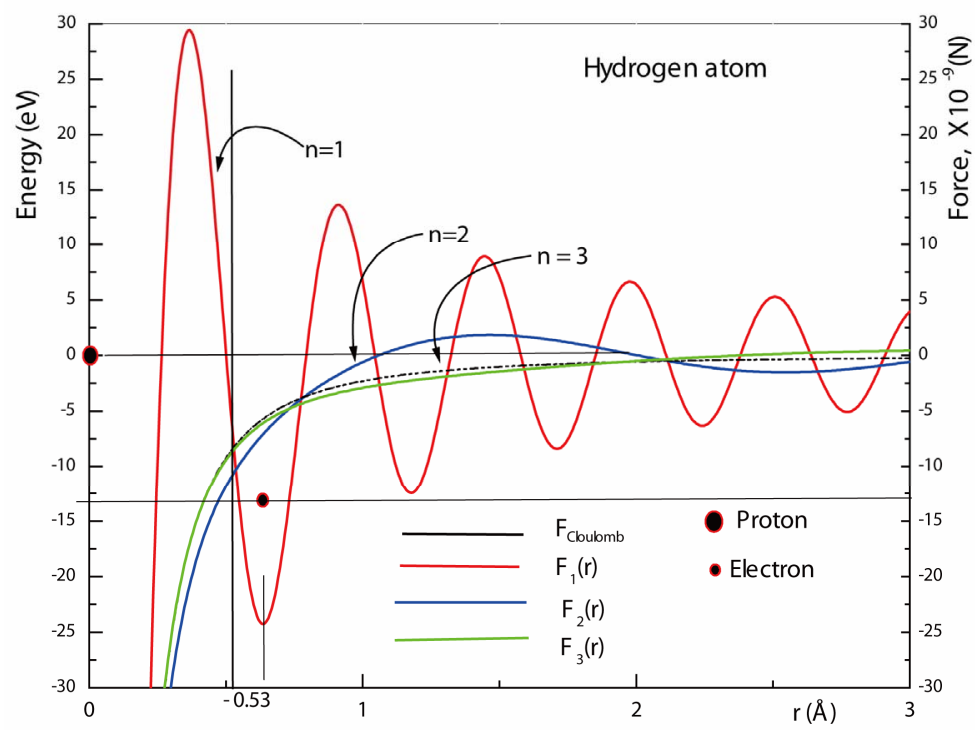

Figure 4. The Coulomb force $F(r)_{\text {Coul }}$ and the interaction oscillatory forces $F_{n}(r)$ between the proton and the electron in the hydrogen atom, for $n=1,2$ and 3.

force between two macroscopic particles are usually performed, using large ionized atoms, for which the escaped electrons come from outer shells of the atoms, making the new electric force to approximate quickly to the classical Coulomb force as indicated in Figure 4.

\section{Conclusion}

We can summarize this work as follows: For the hydrogen atom, the oscillatory potential function is able to predict correctly the values of the total energy and position of the electron in the atom, without the necessity of solving the time-independent Schroedinger wave equa- tion. In addition, it shows several peculiarities of the hydrogen atom, such as: The existence of quantum wells and barriers, forming closed shells around nucleus of the atom. These wells constitute the most probable positions for the electron to occupy when moving around the nucleus of the atom; The emission or absorption of energy by the atom happens when the electron moves from one state of equilibrium to another by tunneling through these potential barriers created by the oscillatory potential; The new potential shows that the electric force between the proton and the electron oscillates, presenting negatives and positives peaks, the positive peaks represent regions where the interaction force between the proton and the 
electron is repulsive and the negative peaks where it is attractive, that is, the electron is always been pushed to the equilibrium position, which is a necessary condition for the stability of the atom. The new potential predicts that de Broglie wavelength associated to the electron is equal to the radius of Bohr $r_{1}=a_{0}$, what is quite different of the condition postulated by Louis de Broglie for the quantization of the angular momentum of the electron in the Bohr Old Quantum Theory. According to the new potential, the electron is not orbiting the nucleus of the atom but oscillating radially, confined by two potential barriers. Whats more, this work presents strong evidences that the Coulomb potential function is not adequate to describe the correct interaction between two microscopic electric charges, because, except at particular points around the nucleus of the atom, it is in disagreement with one of the basic principles of quantum mechanics-the Heisenberg uncertainty principle. The new potential is much more informative than the classical Coulomb potential and more appropriated to accomplish the function for the interaction between two microscopic electric charges.

\section{REFERENCES}

[1] R. P. Feymann, R. B. Leighton and M. Sands, "Lectures on Physics," Addison Wesley, Reading, 1966.

[2] C. Cohen-Tannoudji, B. Diu and F. Laloë, "Quantum Mechanics," John Wiley \& Sons, New York, 1997.

[3] L. Gilder, "The Age of Entanglemen," Alfred A. Knopf, New York, 2008.

[4] W. W. Filho, "Mecânica Quântica," Editora da UFG, Goiânia, 2002.

[5] R. M. Eisberg, "Fundamentals of Modern Physics," John Wiley \& Sons Inc., New York, 1961.

[6] P. A. Tipler, "Modern Physics," Worth Publishers, Inc. Irving, 1986.

[7] L. Pauling and E. B. Wilson, "Introduction to Quantum Mechanics," McGraw Hill, New York, 1935.

[8] W. Hauser, "Introduction to the Principles of Electromagnetism," Addison Wesley Publishing Company Inc., Boston, 1970.

[9] E. Merzbacher, "Quantum Mechanics," John Wiley \& Sons Inc., New York, 1976.

[10] V. A. Fock, "Fundamentals of Quantum Mechanics," Mir Publishers, Moscow, 1982. 